\title{
Collaborative e-Business Process Modelling: Transforming Private EPC to Public BPMN Business Process Models
}

\author{
Volker Hoyer, Eva Bucherer, and Florian Schnabel \\ SAP Research CEC St. Gallen, Switzerland \\ Institute for Media and Communication Management, \\ University of St. Gallen, Switzerland \\ \{volker.hoyer, eva.bucherer, stephan.florian.schnabel\}@sap.com
}

\begin{abstract}
Introducing process orientation to overcome the functionaloriented organizational structure was the main concern within enterprises during the last decade to improve process quality. The next wave of process-oriented enterprises deals with integrating private tasks and processes into cross-organizational business processes characterized by high automation effort and supported by a wide penetration of e-Business technologies. In this work, we propose a concept for transforming internal private processes to publicly visible processes in a semi-automatic way. This transformation will be done by hiding the modelling complexity from the users. Evaluated on the basis of Event-Driven Process Chains (EPC) for private process view and the Business Process Modelling Notation (BPMN) for public process view, this article identifies the challenges both on semantic and syntactic level regarding the integrated mapping between different modelling layers as well as modelling languages.
\end{abstract}

Key words: Collaborative e-Business Process, Event-Driven Process Chain (EPC), Business Process Modelling Notation (BPMN), Business Process Modelling Layers

\section{Introduction}

\subsection{Motivation and Structure}

In the early 1990ies companies focused on the Business Process Reengineering (BPR) approach [1]. Now a changed business environment characterized by borderless enterprises [2] and seamless processes as well as real-time businesses [3] leads to the next wave of process-oriented efficiency improvement. Adaptive Business Networks instead of linear wired value chains spawn new challenges in the context of modelling business processes both on internal (private) and public level. According to popular business opinion, Information Technology (IT) will thereby be transformed into a commodity meaning a common infrastructure like telephone or power grids [4]. On top of a Service-Oriented Architecture (SOA) [5] [6] Business Process Management (BPM) [7] [8] will act as an intermediary between IT infrastructure and strategy layer [9]. It links further to business 
partners processes to create inter-organizational collaboration processes. This will allow enterprises not only to react flexible, on demand and on time, on technical changes but on business environment changes as well.

As shown in previous research activities of the authors [10] the next wave of process orientation is characterized by new challenges regarding private and public e-Business processes. On the one hand process transparency across enterprise borders allows seamless information flows reducing coordination problems. Positive side effects are a flexibility to react to changes of the business environment and economization on financial as well as personnel resources. On the other hand concepts of information hiding are necessary to protect critical internal information, building the foundation for competitive advantages. Additionally, the increasing dynamics in the business environment requires an highly automated transformation between the private and public view on processes to handle a Continuous Improvement Process (CIP). In contrast to the BPR approach [1] the process improvement is achieved by a permanent adaptation and improvement in small steps and not by one radical procedure. In this article, we propose such a transformation concept between private and public business processes that considers the three above-mentioned business challenges and identifies the outcoming challenges both on syntactic and semantic level.

The reminder of the paper is organized as follows. After a brief introduction on electronic collaboration among enterprises in Chapter 2 including the presentation of the business process modelling layer concept (private, public and collaborative), we present in Chapter 3 a two-step transformation concept by means of the business-oriented Event-Driven Process Chains (EPC) on private level and the Business Process Modelling Notation (BPMN) on public level. Based on practical experiences taken in the EU-funded project GENESIS [11], Chapter 4 illustrates identified challenges transforming private and public business processes. An overview about related work in Chapter 5 and a short summary close this work.

\section{Collaborative e-Business Processes}

\section{$2.1 \quad$ E-Business and Collaboration}

During the last years, e-Business has become widely accepted in many different industry segments. By adopting systems that allow for business transactions to be conducted electronically rather than paper-based, enterprises can significantly reduce the effort for data-processing, increase business data accuracy and may even discover new business models or partners [12]. However, due to huge technical complexity and missing globally accepted e-Business standards [13], electronic collaboration is currently limited to portal technology on presentation level. Thereby users are confronted with diverse user interfaces and working processes provided by the business partners (so called 1:n relation). New standardization approaches like ebXML or United Nations Centre for Trade Facilitation and Electronic Business (UN/CEFACT) Core Component Technical Specification (CCTS) [14] try to integrate business processes and semantic aspects on the 
application level [15] enabling real networkability and n:m relation. As a result standardized business processes lead to next generation e-Business frameworks [16] built on SOA and BPM concepts.

In the frame of the EU-funded project GENESIS [11], a consortium of several partners from across Europe proposes such a holistic framework for performing seamless Business-to-Business (B2B) and Business-to-Government (B2G) processes focused on the business environment in Eastern Europe. Typical business processes considered in GENESIS are transactional processes like order, invoice, VAT declaration or bank transfer. The main goal is the research, development and pilot application of the needed methodologies, infrastructure and software components creating a living evolutionary e-Business platform.

\subsection{Business Process Modelling Layers}

Inter-organizational business processes are performed by multiple independent parties. Since organizational borders usually represent boundaries for system interactions and information flows, a number of process particularities arise in comparison to company-internal (private) business processes. To achieve seamless business processes across enterprise borders the heterogeneity of different terminologies and modelling notations used within the organizations have to be overcome. However, autonomy of the different business partners has to be taken into account meaning that an organization should be able to flexibly participate in business relations. Important contributions to handle these challenges with regard to inter-organizational business processes come from workflow management, e.g. the Public-To-Private Approach [17] and the Process-View-Model [18]. As depicted in Figure 1 these approaches distinguish between the internal process (private process) and the cross-organizational interaction (collaborative process).

On private process level, organizations model their internal business processes according to a modelling approach or notation that is most suitable for internal demands independently of the modelling methodologies used by the business partners. In the example shown in Figure 1, enterprise A uses the

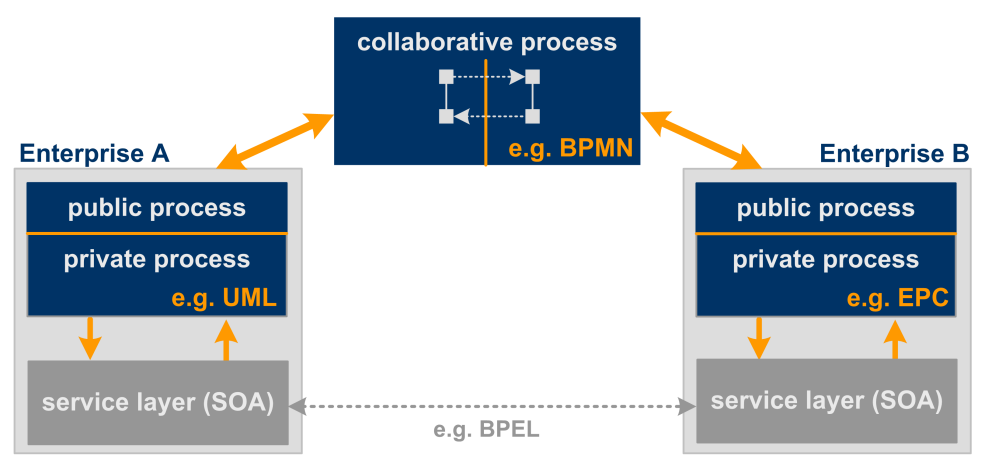

Fig. 1. Business Process Modelling Layers 
Unified Modelling Language (UML) for modelling internal processes whereas enterprise B models with Event-Driven Process Chains (EPC). A comparison addressing the heterogeneity of business process models can be found in [19]. As a response, abstraction concepts hide details of the internal business process from external business partners in the public process view. According to the SOA paradigm [5] public process views are comparable to web service descriptions in the Web Service Definition Language (WSDL) and can be interpreted as a mediator and interface. By hiding the internal process implementation and protecting also critical internal information [20] public process views provided by an organization connect private processes to a collaborative business process. This level defines the interactions of two or more business entities taking place between the defined public processes. One possible language for modelling collaborative processes could be the Business Process Modeling Notation (BPMN) which consolidates ideas from divergent notations into a single standard notation. Examples of notations or methodologies that were reviewed are: UML Activity Diagram, UML EDOC Business Processes, ebXML Business Process Specification Scheme (BPSS), Activity-Descision Flow (ADF) Diagram, RosettaNet, and EPC [21].

Underlying the three presented business process modelling layer a service layer (SOA) represents the technical implementation of the business processes within the enterprises. Thereby, we adhere to the OASIS Reference Model for SOA within this work [6]. It defines SOA as "... a paradigm for organizing and utilizing distributed capabilities that may be under the control of different ownership domains. It provides a uniform means to offer, discover, interact with and use capabilities to produce desired effects consistent with measurable preconditions and expectations." [6]. Especially the widespread SOA implementation Web Services [5] and the respective orchestration language Business Process Execution Language (BPEL) describing the process flow by loose coupling of services play a major role on the executable technical SOA-layer. The mapping of private business processes to BPEL is regarded by several research activities, i.e. EPC/ BPEL [22], BPMN/ BPEL [21], or UML/ BPEL [23].

In the following we abstract from the service layer and focus on the private and public business process layers.

\subsection{Business Process Modelling Languages}

A business process modelling language is an artificial language used to map existing processes or to design new ones. Each modeling language is defined by a set of rules which consists of a syntax (the notation) and a semantic (the meaning) [24].

Both textual and graphical modelling languages exist. In the domain of BPM graphical models are often preferred, as they allow for an overview of the whole process and the presentation of timely and factual interrelations. The decision to employ a specific modelling language should be made in regard to the modelling purpose. Purposes range from pure documentation or auditing to the automation of processes. The construction of a model can reduce the complexity of an actual 
situation as a model usually just represents the relevant aspects. This fact is applied in the idea of hiding private information on a public process level as proposed in this paper.

For an exemplary model transformation we employed extended Event-Driven Process Chains (eEPC) on the private process level. This decision was made with respect to the wide industry dissemination especially in German-speaking regions. The popularity of EPCs is due to their usage within the Architecture for Integrated Information Systems (ARIS) concept [25]. EPCs were developed on the basis of Petri nets during the early 90ies and are especially focused on business and processes. Temporal and logical sequences are described by the use of functions (active elements), events (passive elements), and logical connectors such as "AND", "'OR", "XOR"' to define the control flow. These objects are linked via control flow arcs. By adding additional modelling elements the extended version of EPCs (eEPC) allows in addition for modelling organization and data views.

On the public process level we propose the implementation of the Business Process Modeling Notation (BPMN). BPMN is now a standard administered by the Object Management Group (OMG). It was developed by the Business Process Management Initiative (BPMI) in 2003 as an approach to ease graphical process modelling. It incorporates ideas and experiences of prior standards such as UML activity diagrams and ebXML Business Process Specification Scheme (BPSS). A small number of core elements permit a coherent process modelling. The usage of BPMN for public models is justifiable because of several reasons. According to [7] the basic requirement for collaboration is an open process modelling standard. This aspect disqualifies proprietary standards such as EPC. Apart from being "open" the implemented standard has to be relatively strong so that it is adopted by the industrial community. Applying the workflow pattern of [26] confirms the mightiness of the notation. Also BPMN is a relatively new standard the integration into commercial modelling tools is proceeding quite fast. Furthermore, additional mapping guidelines of BPMN elements to BPEL ease the implementation of BPMN modelled business processes [21]. The use of so-called black and white boxes allows modelling public and private process views.

Especially the last point is one reason to favor BPMN over UML on the public level. UML does not explicitly assist the discrimination of different modelling layers. Furthermore, BPMN is easier to understand for people with a non-technical background.

\section{Transformation Concept}

After building the foundation of this research article the following chapter presents a two-step concept transforming private EPC business processes to public BPMN business processes. 


\subsection{Overview}

As proven in [10] the interrelation between private and public processes is characterized by a high complexity regarding the before-mentioned three business challenges process transparency, information hiding and automated transformation. Additionally, the heterogeneity of the used business process modelling notations requires a concept reducing the complexity for the person modeling processes within the enterprise. Experiences taken in the GENESIS project [11] have demonstrated that especially the employees of Small and Medium-sized Enterprises (SME) characterized by a missing modelling know-how are overcharged to map business processes simultaneously between different modelling views as well as between different modelling notations.

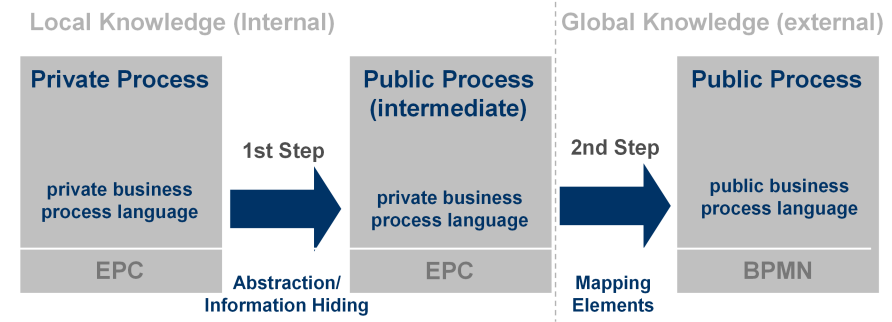

Fig. 2. Two-Step Transformation Concept

To handle these challenges we propose a two-step transformation concept as depicted in Figure 2. The first step abstracts from the private process flows to an intermediate public process layer by using the internal modelling notation. Both process transparency on the one side and information hiding on the other side have to be taken into account in this transformation step. Based on the intermediate layer using the private modelling language (i.e. EPC) the second step maps the abstract public process to the public modelling notation (BPMN). Challenges in this transformation step exist in the differences between the numerous modelling notations using diverse modelling elements, process flow or abstraction representations. An excellent comparison of the two relevant modelling notations in this work, EPC and BPMN, can be found in [27].

\subsection{1st Transformation Step: Abstraction}

As mentioned above, on the private business process layer EPCs are used due to the wide dissemination within the industry. Thereby it can be seen as a placeholder for other adequate modelling notations. By taking into consideration the EPC modelling guidelines [28] the EPC models on private business process layer have to follow the specified EPC rules to apply the EPC abstraction rules of the first transformation step. Figure 3 shows these six abstraction rules which are explained in the following: 
- EPC Abstraction Rule 1. All trivial events have to be eliminated on the public process layer. These passive elements connect the active elements, the functions, and serve as intermediate state allowing a simple understanding for business people. In terms of collaborative e-Business processes these events become no longer necessary.

- EPC Abstraction Rule 2. Initiated events located in the process flow before logical connectors such as "'AND", "'OR"', or "'XOR"' have to be deleted. The process flow including the linked functions is self-explained and sufficient on the public process layer.

- EPC Abstraction Rule 3. Every function of a private process is executed by an assigned organization unit of the enterprise. In consideration of hiding from this internal competitive relevant information, organization units are not visible on public process level.

- EPC Abstraction Rule 4. As mentioned in Chapter 2 collaborative eBusiness processes focus on the information exchange. Only functions sending or receiving a message (data object) are part of the public business process layer. All other functions have to be dropped.

- EPC Abstraction Rule 5. Sensitive critical process steps not interacting directly with external business partners have to be abstracted by means of

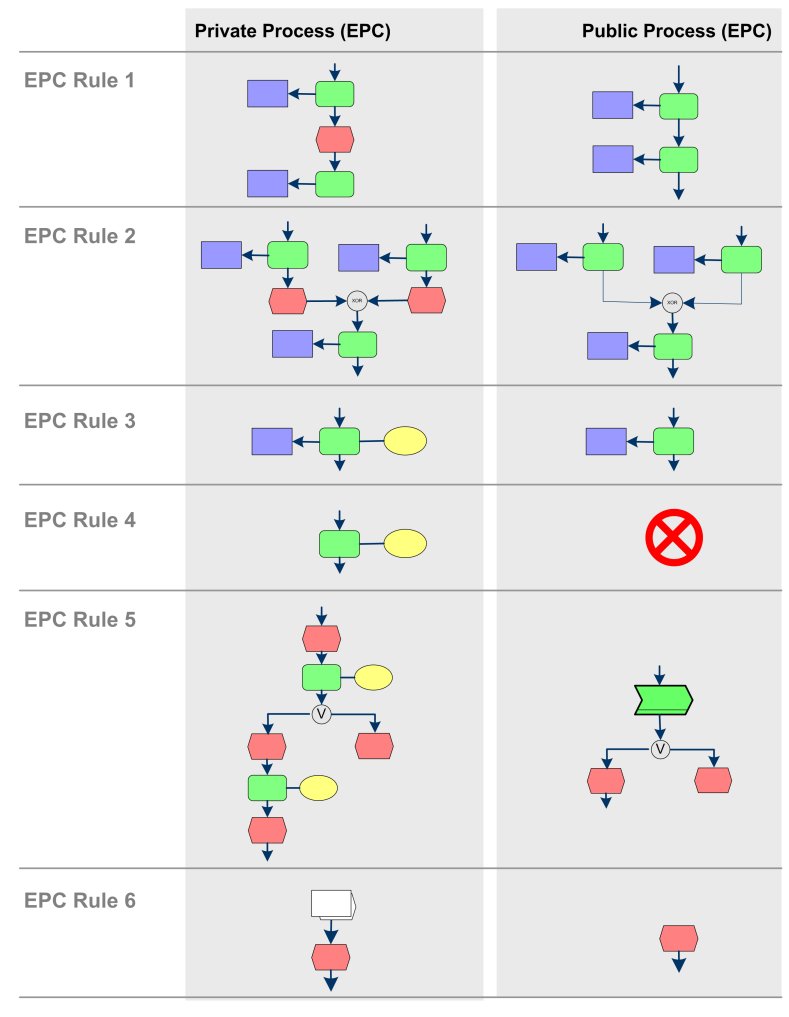

Fig. 3. EPC Abstraction Rules (1st Transformation Step) 
process modules. Public relevant events have to follow the process modules to describe the public process flow.

- EPC Abstraction Rule 6. Process interfaces can be used to point from a function to a refining sub-process. This hierarchical representation does not exist on the public process layer.

\subsection{2nd Transformation Step: Mapping to the public business process notation}

On basis of the intermediate layer abstracting from the private business process and using the internal process modelling notation (in case of this work EPC) the second step maps to the public business process notation. Reasons for the approprioateness of BPMN were evaluated above.

- Mapping Rule 1. According to the semantic meaning of the EPC events the connectors in BPMN have to be added with constraints to represent the conditioned process flow.

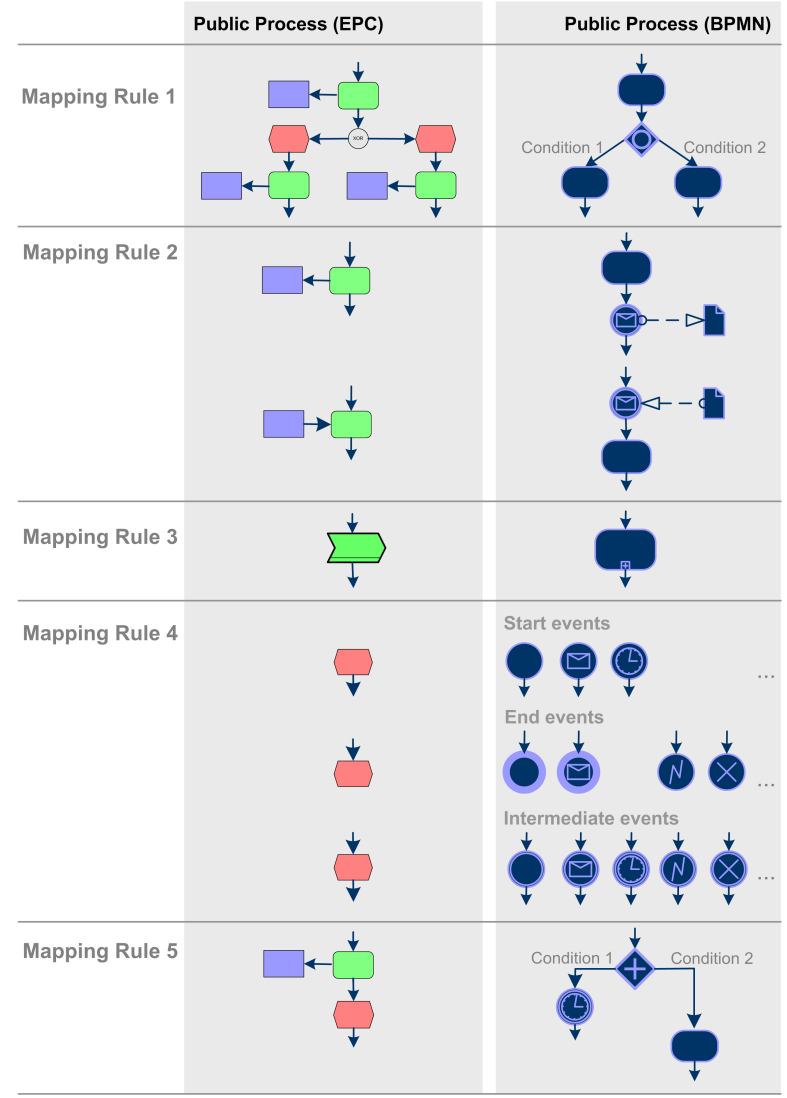

Fig. 4. Mapping Rules (2nd Transformation Step) 
- Mapping Rule 2. In addition to the sending or receiving EPC functions the BPMN message element has to follow the functions to play by the modelling rules of BPMN.

- Mapping Rule 3. EPC process modules which abstract from internal process flows correspond to BPMN sub-functions. Therefore they can be directly mapped and hidden from internal process steps like a black box.

- Mapping Rule 4. According to their semantic meaning EPC events have to be transfered to BPMN events. In contrast to the EPC notation with only one event type there exist three basic event types in BPMN (start, intermediate and end events) with several sub types like message, timer, rule, link, multiple, error, cancel, or compensation.

- Mapping Rule 5. Semantic related functions and events on private level have to be transfered to an adequate BPMN representation.

\section{Challenges}

The combination of the two transformation steps leads from private EPC to public BPMN business process models. The envisioned bi-directional process interfaces can not be achieved completely due to the different representation types and granularity of the two modelling languages. But these syntactic challenges (i.e. mapping rule 1) play only a tangential role due to the fact that the introduced intermediate layer minimizes such syntactic differences.

The actual large challenges exist on semantic level of the second transformation step. As already indicated event mapping between the two modelling notations is limited to uni-directional relation (mapping rule 4) [29] and requires additional user interactions. Another challenge is related to a slight variance of semantic representation of a process flow as can be seen by mapping rule 5 . Experiences taken in the GENESIS project have demonstrated how unavoidable user interactions lead to a wide variance of process interpretation. Without standardized mapping guidelines to generate a common understanding public processes will not be identically interpreted by the users and will not allow a flexible collaboration. Finally the users decide about success or failure of a concept [10]. That is why these identified semantic challenges have to solve with only a low number of "semantic mapping rules".

\section{Related Work}

In literature modelling collaborative e-Business processes is discussed from several perspectives. Existing approaches are mostly limited to either private, public or collaborative processes like [18], [30], or [17].

A methodology dealing with collaborative processes is the UN/CEFACT Modelling Methodology (UMM) [31]. Based on Unified Modelling Language (UML) and Rational Unified Process (RUP) UMM is a methodology similar to a software process and supports components to capture business process knowledge. According to the Open-EDI reference model [32] the UMM specifies 
collaborative business processes involving information exchange in a technologyneutral, implementation-independent manner. It combines an integrated process (UML) and data (Core Component Technical Specification, CCTS) modelling approach [14] as well as modelling business collaboration in context [33].

An approach focusing on modelling inter-organizational processes is described in [34]. According to [35] the coordination between the different organizations requires an agreement on how to interact and exchange information. Therefore a public process model is built detailing the process interfaces between the organizations. In a further step private processes are aligned to the public process. [34] assume an existing public process the private processes could be aligned to. In contrast the approach of this paper will derive a public process out of private processes.

Another approach on inter-organizational interoperability is described by [29]. It states that the partners' private processes modelled in different notations have to be integrated into one collaborative process model. This is done by a model transformation via an intermediate representation. [36] define an intermediate representation as a model which is exported into a standard form. In [29] the model transformation via an intermediate representation is revealed by an example of a horizontal model transformation. A horizontal transformation means the transformation on the same abstraction layer. As realization of a horizontal transformation a XML-based mapping of an eEPC model to a BPMN model is regarded. Based on the graphically modelled private processes a public view is created and exported into a XML-based intermediate representation. Such an intermediate representation is the EPC Markup Language (EPML) for EPCs and the BPMN Markup Language (BNML) for BPMN. In [29] the goal notation for the collaborative process is BPMN. Therefore the models in EPML notation are transformed into the BNML using a XSLT-script. The resulting process in BNML notation can then be transformed into a BPMN model. The last step of the transformation is to manually combine the public processes to a collaborative process.

\section{Conclusion and Further Work}

In the frame of this article, a transformation concept is presented introducing an intermediate layer to map private business processes to public business processes by means of the two modelling languages EPC and BPMN. Taking into account the two challenges process transparency on the one side and information hiding on the other side the concept reduces the complexity users are facing with regard to modelling collaborative e-Business processes across enterprises. First experiences taken in the GENESIS project have proven the applicability on conceptual level.

Further work will deal with the implementation of a script transforming the private and public business processes in a semi-automatic manner eliminating manual user activities that leads to work more on strategic issues and thus increase the value-added productivity [10]. Also, the investigation about how 
much transformation work could be freed from the user will be part of further research activities to clarify the saving potential in terms of manual activities as well as time aspects.

Acknowledgments. This paper has been created closely to research activities during the EU-funded project GENESIS (Contract Number FP6-027867) [11].

\section{References}

1. Hammer, M., Champy, J.: Reengineering the corporation. Brealey, London (1993)

2. Picot, A., Reichwald, R., Wigand, R.T.: Information Organization and Management: Expanding Markets and Corporate Boundaries. John Wiley and Sons, New York (1999)

3. Alt, R., Oesterle, H.: Real-Time Business. Springer, Berlin et al. (2004)

4. Carr, N.G.: IT doesn't matter. Harvard Business Review 81(5) (2003) 41-49

5. Alonso, G., Casati, F., Kuno, H., Machiraju, V.: Web Services Concepts, Architectures and Applications. Springer, Berlin et al. (2004)

6. McKenzie, C.M., Laskey, K., McCabe, F., Brown, P.F., Metz, R.: Reference model for service oriented architecture 1.0. http://www.oasisopen.org/committees/download.php/19679/soa-rm-cs.pdf (2006)

7. Smith, H., Fingar, P.: Business process management: the third wave. MeghanKiffer Press, Tampa, Fla. (2003)

8. Harrington, H.: Business Process Improvement: The Breakthrough Strategy for Total Quality, Productivity, and Competitiveness. McGraw-Hill, New York (1991)

9. Oesterle, H., Back, A., Winter, R.: Business Engineering. Springer, Berlin et al. (2004)

10. Hoyer, V., Christ, O.: Collaborative e-business process modelling: A holistic analysis framework focused on small and medium-sized enterprises. Lecture Notes in Computer Science 4439 (2007) 41-53

11. GENESIS: EU project GENESIS (FP6-027867). http://www.genesis-ist.eu (2007)

12. United Nations Economic Commission for Europe: A roadmap towards paperless trade. http://www.unece.org/cefact (2006)

13. Zhao, K., Xia, M., Shaw, M.J.: Vertical e-business standards and standards developing organizations. Electronic Markets 15(4) (2005) 289-300

14. UN/CEFACT: Core component technical specification v2.01. http://www.untmg.org (2003)

15. Janner, T., Schmidt, A., Schroth, C., Stuhec, G.: From EDI to UN/CEFACT: An evolutionary path towards a next generation e-business framework. In: Proceedings of the 5th International Conference on e-Business (NCEB2006). (2006)

16. Hoyer, V., Janner, T., Mayer, P., Raus, M., Schroth, C.: Small and medium enterprise's benefits of next generation e-business platforms. The Business Review, Cambridge (BRC) 6(1) (2006) 285-291

17. van der Aalst, W.M.P., Weske, M.: The P2P approach to interorganizational workflows. Lecture Notes in Computer Science 2068 (2001) 140-156

18. Shen, M., Liu, D.R.: Coordinating interorganizational workflows based on processviews. In: Proceedings of the 12th International Conference on Database and Expert Systems Applications (DEXA'01). (2001) 274-283

19. Mendling, J., Neumann, G., Nuettgens, M.: A comparison of XML interchange format for business process modelling. In: Proceedings of EMISA 2004 - Information Systems in E-Business and E-Government. (2004) 
20. Parnas, D.L.: On the criteria to be used in decomposing systems into modules. Communications of the ACM 15(12) (1972) 1053-1058

21. Object Management Group: Business process modeling notation specification, OMG final adopted specification. http://www.bpmn.org (2006)

22. Mendling, J., Ziemann, J.: Transformation of BPEL processes to EPCs. In: Proceedings of the 4th GI Workshop. (2005)

23. Hofreiter, B., Huemer, C.: Transforming UMM business collaboration models to BPEL. Lecture Notes in Computer Science 3292 (2004) 507-519

24. Harel, D., Rumpe, B.: Modeling languages: Syntax, semantics and all that stuff. technical paper number mcs00-16, the weizmann institute of science (2000)

25. Scheer, A.W.: ARIS, Business Process Frameworks. Springer, Berlin et al. (1999)

26. van der Aalst, W.M.P., ter Hofstede, A.H.M., Kiepuszewski, B., Barros, A.P.: Workflow pattern. Distributed and Parallel Databases 14(3) (2003) 5-51

27. Vanderhaegen, D., Zang, S., Scheer, A.W.: Inter-organizational process management through model transformation. working paper 182, university of saarbruecken, institute for information systems, http://www.iwi.unisb.de/download/iwihefte/iwiheft_178.pdf (2005)

28. IDS-Scheer: ARIS Method Version 7.0. IDS-Scheer (2005)

29. Vanderhaeghen, D., Zang, S., Hofer, A., Adam, O.: XML-based transformation of business process models - enabler for collaborative business process management. (In: Proceedings of 11 th Conference)

30. Medjahed, B., Benatallah, B., Bouguettaya, A., Ngu, A., Elmagarmid, A.: Business-to-business interactions issues. The VLDB Journal (2003) 12: 59-85 / Digital Object Identifier (DOI) 10.1007/s00778-003-0087-z 12 (2003) 59-85

31. UN/CEFACT: UN/CEFACT Modeling methodology (UMM). http://www.unece.org/cefact/umm (2006)

32. ISO/IEC: Information technology - Open-EDI reference model, ISO/IEC standard 14662:2004(e). http://www.iso.org (2004)

33. Hofreiter, B., Huemer, C.: Modeling business collaborations in context. Lecture Notes in Computer Science 2889 (2003) 829-844

34. Legner, C., Vogel, T., Loehe, J., Mayerl, C.: Transforming inter-organizational business processes into service-oriented architectures - method and application in the automotive industry. In: VDE Verlag. (2007)

35. ATHENA: Cross-organizational business process requirements and the state-ofthe-art in research, technology and standards. (2005)

36. Sendall, S., Kozaczynski, W.: Model transformation: the heart and soul of modeldriven software development. Software (IEEE) 20(5) (2003) 42-45 